\title{
BMJ Open Effects of interpregnancy interval on pregnancy complications: protocol for systematic review and meta-analysis
}

\author{
Amanuel Tesfay Gebremedhin, ${ }^{1}$ Annette K Regan, ${ }^{1}$ Eva Malacova, ${ }^{1}$ \\ M Luke Marinovich, ${ }^{1}$ Stephen Ball, ${ }^{2}$ Damien Foo, ${ }^{1}$ Gavin Pereira ${ }^{1}$
}

To cite: Gebremedhin AT, Regan AK, Malacova E, et al. Effects of interpregnancy interval on pregnancy complications: protocol for systematic review and meta-analysis. BMJ Open 2018;8:e025008. doi:10.1136/ bmjopen-2018-025008

- Prepublication history and additional material for this paper are available online. To view please visit the journal (http:// dx.doi.org/10.1136/bmjopen2018-025008).

Received 25 June 2018

Revised 11 July 2018

Accepted 12 July 2018

D Check for updates

C C Author(s) (or their employer(s)) 2018. Re-use permitted under CC BY-NC. No commercial re-use. See rights and permissions. Published by BMJ.

${ }^{1}$ School of Public Health, Curtin University, Perth, Western Australia, Australia

${ }^{2}$ Pre-Hospital, Resuscitation and Emergency Care Research Unit (PRECRU), School of Nursing, Midwifery and Paramedicine, Curtin University, Perth, Western Australia, Australia

Correspondence to

Mr Amanuel

Tesfay Gebremedhin; a.gebremedhin1@postgrad. curtin.edu.au

\section{ABSTRACT}

Introduction Interpregnancy interval (IPI) is the length of time between a birth and conception of the next pregnancy. Evidence suggests that both short and long IPIs are at increased risk of adverse pregnancy and perinatal outcomes. Relatively less attention has been directed towards investigating the effect of IPI on pregnancy complications, and the studies that have been conducted have shown mixed results. This systematic review will aim to provide an update to the most recent available evidence on the effect of IPI on pregnancy complications. Method and analysis We will search electronic databases such as Ovid/MEDLINE, EMBASE, CINAHL, Scopus, Web of Science and PubMed to identify peerreviewed articles on the effects of IPI on pregnancy complications. We will include articles published from start of indexing until 12 February 2018 without any restriction to geographic setting. We will limit the search to literature published in English language and human subjects. Two independent reviewers will screen titles and abstracts and select full-text articles that meet the eligibility criteria. The Newcastle-Ottawa tool will be used to assess quality of observational studies. Where data permit, meta-analyses will be performed for individual pregnancy complications. A subgroup analyses by country categories (high-income vs low and middle-income countries) based on World Bank income group will be performed. Where meta-analysis is not possible, we will provide a description of data without further attempt to quantitatively pool results.

Ethics and dissemination Formal ethical approval is not required as primary data will not be collected. The results will be published in peer-reviewed journals and presented at national and international conferences.

PROSPERO registration number CRD42018088578.

\section{INTRODUCTION}

The length of time between birth and the beginning of the following pregnancy (interpregnancy interval (IPI)) has been linked to an increased risk of adverse outcomes in infants and their mothers. ${ }^{1-4}$ To reduce this risk, the WHO and the American College of Obstetrics and Gynaecology suggest an interval of at least 2 years and a minimum of 18 months following a live birth, respectively. ${ }^{23}$ IPI is viewed as a potential modifiable

\section{Strengths and limitations of this study}

The proposed systematic review and meta-analysis will adhere to the Preferred Reporting Items fo Systematic Reviews and Meta Analyses guidelines.

- The review aims to provide an update to the most recent available evidence on the effect of interpregnancy interval on pregnancy complications.

- Two independent reviewers will screen titles and abstracts, study eligibility and perform the quality assessment.

- This review will only include the published literature in the English language.

risk factor for adverse maternal and perinatal outcomes for planned pregnancies.

The importance of birth spacing has been a focus for perinatal researchers and policy-makers for nearly a century. ${ }^{5}$ Studies have revealed that both short and long IPIs are potentially associated with increased risk of adverse perinatal outcomes, including stillbirth, small for gestational age, preterm delivery and neonatal death. ${ }^{1346}$ Conversely, the effect of IPI on complications during pregnancy has received less attention.

There is a growing body of literature that recognises the association between short IPIs and risk of premature rupture of membrane $(\mathrm{PROM}),{ }^{78}$ placental abruption, placenta praevia, ${ }^{9}$ uterine rupture for women who previously delivered by caesarean section ${ }^{10} 11$ and gestational diabetes. ${ }^{12}$ Similarly, long IPIs have long been associated with increased risk of pre-eclampsia ${ }^{1314}$ and labour dystocia. ${ }^{4}$

Although previous reviews ${ }^{15}$ have suggested that IPI is associated with risk of pregnancy complications, these reviews did not identify a sufficient number of studies to evaluate the effect of IPI on pregnancy complications.

The two systematic reviews investigating the effect of IPI on maternal health/outcomes were published 10 years and 5 years ago, 
respectively, ${ }^{15}$ and there has since been increasing attention paid to this area and a number of publications. ${ }^{12}$ 16-19 Meanwhile, the reviews have been either limited to few maternal outcomes of interest (ie, maternal haemorrhage, PROM $)^{9}$ or not included results from studies published in the last decade. ${ }^{1}$ A further systematic review of the effect of IPI on pregnancy complications is warranted, with a view to meta-analysis of the outcomes.

This systematic review will explore the effect of IPI on pregnancy complications. The information obtained from this review is important to inform women, their family and clinicians regarding IPI. The main purpose of the systematic review is to update, compile and critically review the evidence on the effects of IPI on pregnancy complications.

\section{METHODS AND DESIGN Population}

The systematic review will include multiparous women with information on length of interval between two consecutive pregnancies. We will not exclude studies that implemented restrictions on age, ethnic group, parity and socioeconomic status.

\section{Study design}

This systematic review will include all observational prospective or retrospective studies that have assessed the effects of IPI with various pregnancy complications according to birth interval categories. Randomised controlled trials (RCT) are unlikely to be identified due to exposure of interest but will be included if available.

\section{Comparator(s)/control}

When assessed as a categorical variable, the reference IPI category will be 18-23 months.

\section{Outcomes}

The outcomes of interest in this review are pregnancy complications, defined as gestational diabetes, gestational hypertension, pre-eclampsia, uterine rupture, placental abruption, placenta praevia, PROM and labour dystocia.

\section{DATA SOURCES AND SEARCH STRATEGY}

We will conduct electronic searches in Ovid/MEDLINE, EMBASE, CINAHL, Scopus, Web of Science and PubMed databases, using a combination of medical subject headings (MeSH) and keywords related to IPI and pregnancy complications. We will include articles published from start of indexing until 12 February 2018 without any restriction on study type or geographic setting. A search strategy was developed (see table 1 for search criteria and online Supplementary file 1 for detailed search strategy for each database).

The search strategy will be piloted across each database to improve the effectiveness of the final search. We will also check the reference list of primary studies that will be selected for full-text evaluation for additional potentially

\begin{tabular}{|c|c|}
\hline & Search terms \\
\hline $\begin{array}{l}\text { Interpregnancy } \\
\text { interval }\end{array}$ & $\begin{array}{l}\text { Subject heading (MeSH) term: Birth interval } \\
\text { Keywords: '“birth interval' or “*birth } \\
\text { spacing' or '*conception interval' or } \\
\text { '*conception spacing' or '*delivery interval' } \\
\text { or '*delivery spacing' or '*pregnancy } \\
\text { interval' or '*pregnancy spacing' }\end{array}$ \\
\hline $\begin{array}{l}\text { Pregnancy } \\
\text { complications }\end{array}$ & $\begin{array}{l}\text { Subject heading (MeSH) term: 'Pregnancy } \\
\text { Complications' } \\
\text { Keywords: 'obstetric complication"' or } \\
\text { 'maternal complication*' or 'maternal } \\
\text { morbidit"' or 'maternal outcome*' }\end{array}$ \\
\hline
\end{tabular}

relevant studies not identified by the electronic search. We will include studies published in peer-reviewed journals conducted with human populations and restricted to English language. Corresponding authors will be contacted to request information not presented in the manuscripts that are required for the review.

\section{ELIGIBILITY CRITERIA Inclusion criteria}

The studies to be included in this review are required to fulfil two criteria.

Study design criterion: all observational studies evaluating the association between IPI and pregnancy complications.

Exposure criterion: studies that investigate IPI or birth interval as the primary exposure. IPI is defined as the length of time between the end of a pregnancy and the start of the next pregnancy. Birth interval is defined as the time elapsed between the end of one pregnancy and the end of the next pregnancy.

\section{Exclusion criteria}

Studies will be excluded based on three criteria. (1) Non-primary studies: case series or reports, editorials, letters to the editor or reviews without original data. (2) Studies with insufficient information on adjusted effect (eg, unclear adjustment variable, missing CI estimates). (3) Studies that do not investigate IPI as a primary exposure.

\section{Study selection process and software}

All unique studies identified from each electronic database will be imported into an EndNote library. For reproducibility and to expedite a future update of the review, this library will be published as online Supplementary data. Further screening of titles and abstracts will be accomplished by two independent investigators. Results will be stored using Covidence, a web-based software tool that (1) allows collation of search results, (2) screen abstracts and full text articles, (3) extract data from selected articles, (4) conduct risk of bias assessment and (5) resolve disagreements and export data. In accordance with Preferred Reporting Items for Systematic Reviews 
and Meta-Analyses (PRISMA), a flow diagram will be used to report the screening process. From the set of studies screened by title and abstract, two reviewers will independently screen full-text articles based on the eligibility criteria. Any discrepancies between the two reviewers for studies that have been included or excluded will be discussed first, if an agreement cannot be reached, a third investigator will be consulted for moderation. The reason for excluding each study will also be recorded.

\section{Risk of bias (quality) assessment}

The quality of included studies will be assessed by two independent reviewers using The Newcastle-Ottawa Scale for assessing quality of cohort and cross-sectional studies. ${ }^{20}$ Any disagreement which arises between the reviewers will be resolved through discussion with a third reviewer.

\section{Data extraction}

Data will be extracted from all included studies by two independent reviewers using a specifically developed data extraction form in line with the eligibility criteria and outcomes of interest. For each study, the following data will be extracted (1) author names, (2) publication year, (3) study period, (4) geographic location, (5) World Bank income category (at the time of publication), (6) study design, (7) sample size, (8) exposure, (9) outcome measure of interest, (10) adjustment or matching variables, (11) effect size and (12) response rate (where indicated).

\section{Data synthesis and analysis}

The final review will include data presented in summary tables and a narrative synthesis to describe the variables listed in the data extraction section. Where data permit, meta-analyses will be performed for individual pregnancy complications. We will apply random effects meta-analysis using the generic inverse variance method to explore the association between IPIs and pregnancy complications. ${ }^{21}{ }^{22}$ We will calculate pooled odss ratio (OR) from all studies that provided adjusted OR or risk ratio with 95\% CIs for each pregnancy complication (outcome of interest). Egger's weighted regression test will be used to assess publication bias. ${ }^{23}$ The $\mathrm{I}^{2}$ statistic will be reported as a measure of heterogeneity between studies. ${ }^{24}$ Where meta-analysis is not possible, we will present data without quantitatively synthesising it. If the same data are presented in multiple studies, then those providing the most information will be considered.

\section{Subgroup analyses}

Subgroup analyses by country categories based on World Bank income group (high-income countries vs low and middle-income countries) will be performed.

\section{Confidence in cumulative evidence}

The quality of the findings on each outcome of interest across studies will be assessed using Grading of Recommendations, Assessment, Development and Evaluations
(GRADE) guidelines, which are developed by the GRADE Working Group. ${ }^{25}$ The GRADE approach will allow us to determine the quality of the evidence of each outcome. The GRADE system classifies the quality of evidence as very low (very uncertain effect estimates), low (further research will likely change the effect estimate), moderate (further research may change the estimate and our confidence in it) or high (further research is very unlikely to change our confidence in the estimate of effect).

\section{Patient and public involvement}

Members of the community Healthy Pregnancies Consumer Reference Group will provide community and consumer perspectives to this study. This group will provide an insight into issues that affect their pregnancy planning decisions, contextualise results and provide participant experience.

\section{Ethics and dissemination}

Formal ethical approval is not required as primary data will not be collected. This protocol adheres to the PRISMA protocols guidelines. ${ }^{26}$ In addition, the findings of the systematic review will be reported according to the PRISMA statement. ${ }^{27}$

\section{Review registration}

This review has been registered with International Prospective Register for Systematic Reviews (PROSPERO) under the identification code: CRD42018088578.

\section{Updates to study protocol}

If any updates to the study protocol are required, these will be listed and included as supplementary information along with a final manuscript and updated on the PROSPERO register.

\section{DISCUSSION}

Families want to know the best time at which they conceive their next child in order to have a safer pregnancy and healthy baby. Clinicians need evidence-based recommendations to provide advice on the optimal IPI leading to fewer maternal and perinatal complications. For planned pregnancies, IPI is modifiable, and such recommendations may therefore be useful for preventing adverse maternal/pregnancy outcomes. The current WHO recommendations, which suggest that women wait at least 2 years after delivering a live birth, ${ }^{2}$ were based on a review of observational studies predominantly in low-income and middle-income populations, which may not be generalisable to high-income countries. Context specific and updated evidence is warranted to clarify whether the evidence of studies investigated the effect of IPI on pregnancy complications is sufficient for decision-making.

This will be a comprehensive systematic review investigating the effect of IPI on pregnancy complications. Previous reviews have been limited to few maternal outcome of interest ${ }^{15}$ or have not included results from studies published in the last 10 years. ${ }^{1}$ A systematic review 
investigating effect of IPI on pregnancy complications is now warranted. Systematic documentation and synthesising of literature on the effect of IPI on various pregnancy complications will be important to set and revise evidence-based guidelines for IPIs. By updating the current state of knowledge in IPI research, this review will provide a basis for guiding future studies and future global policies for family planning.

Acknowledgements We are very grateful for the expert assistance of Faculty of Health Sciences librarian, Ms Diana Blackwood.

Contributors ATG, AR, GP and EM conceived the idea, planned and designed the study protocol. ATG wrote the first draft. DF, SB and LM contributed to the development of the protocol and manuscript. All authors contributed to the initial question development, search strategy, study selection criteria and have approved and contributed to the final written manuscript.

Funding ATG is recipient of a Curtin International Postgraduate Research Scholarship (CIPRS). GP is supported by National Health and Medical Research Council project grants (GNT1099655, GNT1141510). AR is supported by a National Health and Medical Research Council fellowship (GNT1138425).

Competing interests None declared.

Patient consent Not required.

Provenance and peer review Not commissioned; peer reviewed for ethical and funding approval prior to submission.

Data sharing statement Supporting data can be found at the International prospective Register of Systematic Reviews (PROSPERO) website, with registration number CRD42018088578.

Open access This is an open access article distributed in accordance with the Creative Commons Attribution Non Commercial (CC BY-NC 4.0) license, which permits others to distribute, remix, adapt, build upon this work non-commercially, and license their derivative works on different terms, provided the original work is properly cited, appropriate credit is given, any changes made indicated, and the use is non-commercial. See: http://creativecommons.org/licenses/by-nc/4.0/.

\section{REFERENCES}

1. Conde-Agudelo A, Rosas-Bermúdez A, Kafury-Goeta AC. Effects of birth spacing on maternal health: a systematic review. Am J Obstet Gynecol 2007;196:297-308.

2. World Health Organization. Report of a WHO technical consultation on birth spacing [Internet]. Geneva Switzerland 13-15 June 2005. 2005:44 http://www.who.int/maternal_child_adolescent/documents/ birth_spacing05/en/ (cited 5 Jan 2017).

3. Shachar BZ, Lyell DJ. Interpregnancy interval and obstetrical complications. Obstet Gynecol Surv 2012;67:584-96.

4. Zhu BP, Rolfs RT, Nangle BE, et al. Effect of the interval between pregnancies on perinatal outcomes. N Engl J Med 1999;340:589-94.

5. Winikoff B. The effects of birth spacing on child and maternal health. Stud Fam Plann 1983;14:231-45.

6. Eastman NJ. The effect of the interval between births on maternal and fetal outlook. Am J Obstet Gynecol 1944;47:445-66.
7. Getahun D, Strickland D, Ananth CV, et al. Recurrence of preterm premature rupture of membranes in relation to interval between pregnancies. Am J Obstet Gynecol 2010;202:570.e1-6.

8. Razzaque A, Da Vanzo J, Rahman M, et al. Pregnancy spacing and maternal morbidity in Matlab, Bangladesh. Int J Gynaecol Obstet 2005;89(Suppl 1):S41-S49.

9. Getahun D, Oyelese Y, Salihu HM, et al. Previous cesarean delivery and risks of placenta previa and placental abruption. Obstet Gynecol 2006;107:771-8.

10. Bujold E, Gauthier RJ. Risk of uterine rupture associated with an interdelivery interval between 18 and 24 months. Obstet Gynecol 2010;115:1003-6.

11. Esposito MA, Menihan CA, Malee MP. Association of interpregnancy interval with uterine scar failure in labor: a case-control study. $A m \mathrm{~J}$ Obstet Gynecol 2000;183:1180-3.

12. Hanley GE, Hutcheon JA, Kinniburgh BA, et al. Interpregnancy interval and adverse pregnancy outcomes: an analysis of successive pregnancies. Obstet Gynecol 2017;129:408-15.

13. Harutyunyan A, Armenian $\mathrm{H}$, Petrosyan V. Interbirth interval and history of previous preeclampsia: a case-control study among multiparous women. BMC Pregnancy Childbirth 2013;13:244.

14. Basso O, Christensen $\mathrm{K}$, Olsen J. Higher risk of pre-eclampsia after change of partner. An effect of longer interpregnancy intervals? Epidemiology 2001;12:624-9.

15. Wendt A, Gibbs CM, Peters S, et al. Impact of increasing interpregnancy interval on maternal and infant health. Paediatr Perinat Epidemiol 2012;26(Suppl 1):239-58.

16. Mignini LE, Carroli G, Betran AP, et al. Interpregnancy interval and perinatal outcomes across Latin America from 1990 to 2009: a large multi-country study. BJOG 2016;123:730-7.

17. Cecatti JG, Correa-Silva EPB, Milanez $\mathrm{H}$, et al. The associations between inter-pregnancy interval and maternal and neonatal outcomes in Brazil. Matern Child Health J 2008;12:275-81.

18. Kessous $R$, Sheiner $E$. Is there an association between short interval from previous cesarean section and adverse obstetric and perinatal outcome? J Matern Fetal Neonatal Med 2013;26:1003-6.

19. Appareddy S, Pryor J, Bailey B. Inter-pregnancy interval and adverse outcomes: evidence for an additional risk in health disparate populations. The Journal of Maternal-Fetal \& Neonatal Medicine 2017;30:2640-4.

20. Wells G, Shea B, O'connell D, et al. The Newcastle-Ottawa Scale (NOS) for assessing the quality of nonrandomised studies in metaanalyses. Oxford: Ottawa Hospital Research Institute, 2015.

21. Stijnen T, Hamza TH, Ozdemir P. Random effects metaanalysis of event outcome in the framework of the generalized linear mixed model with applications in sparse data. Stat Med 2010;29:3046-67.

22. DerSimonian R, Kacker R. Random-effects model for meta-analysis of clinical trials: an update. Contemp Clin Trials 2007;28:105-14.

23. Ahmed I, Sutton AJ, Riley RD. Assessment of publication bias, selection bias, and unavailable data in meta-analyses using individual participant data: a database survey. BMJ 2012;344:d7762.

24. Higgins JPT. Cochrane Handbook for Systematic Reviews of Interventions. 1 edn. Hoboken: Wiley, 2008.

25. Balshem $\mathrm{H}$, Helfand $\mathrm{M}$, Schünemann $\mathrm{HJ}$, et al. GRADE guidelines: 3 . Rating the quality of evidence. J Clin Epidemiol 2011;64:401-6.

26. Moher D, Shamseer L, Clarke M, et al. Preferred reporting items for systematic review and meta-analysis protocols (PRISMA-P) 2015 statement. Syst Rev 2015;4:1.

27. Moher D, Liberati A, Tetzlaff J, et al. Preferred reporting items for systematic reviews and meta-analyses: the PRISMA statement. Ann Intern Med 2009;151:264. 